\title{
Merging of galaxies with central black holes II. Evolution of the black hole binary and the structure of the core
}

\author{
Junichiro Makino \\ Department of Graphics and Computer Science, \\ College of Arts and Sciences, University of Tokyo, \\ 3-8-1 Komaba, Meguro-ku, Tokyo 153, Japan.
}

\begin{abstract}
We investigated the evolution of the black hole binary formed by the merging of two galaxies each containing a central massive black hole. Our main goal here is to determine if the black hole binary can merge through the hardening by dynamical friction and the gravitational wave radiation. We performed $N$-body simulations of merging of two galaxies with wide range of total number of particles to investigate the effect of the number of particles on the evolution of the black hole binary. We found that the evolution timescale was independent of the number of particles in the galaxy $N$ until the separation reaches a critical value. After the separation became smaller than this critical value, the evolution timescale was longer for larger number of particles. Qualitatively, this behavior is understood naturally the result the "loss-cone" effect. However, the dependence of the timescale on $N$ is noticeably weaker than the theoretical prediction. In addition, the critical separation is smaller than the theoretical prediction. The timescale of evolution through gravitational radiation at this critical separation is longer than the Hubble time. We discuss the reason of these discrepancy and the implication of the present result on the structure of the ellipticals and QSO activities.
\end{abstract}

subject headings: galaxies:interactions, galaxies:nuclei. 


\section{Introduction}

The possibility of the formation of massive black hole binaries at the cores of the elliptical galaxies was first pointed out by Begelman, et al. (1980, hereafter BBR). Their argument is summarized as follows. The most likely energy source for AGN or QSO activities is the massive black holes with the mass $M_{\mathrm{BH}} \simeq 10^{8} M_{\odot}$. If two galaxies with central massive black holes merge with each other, the BHs will sink to the center of the merger remnant because of the dynamical friction from stars, and form a binary.

BBR argued that such a binary would have the typical lifetime larger than the Hubble time, since the BH binary would create the "loss cone" in the distribution of field particles, which is repopulated only in the relaxation timescale of the core. According to their argument, once the separation of the binary comes down to the loss-cone radius, $r_{\mathrm{lc}}$, which is given by

$$
r_{\mathrm{lc}}=\left(\frac{M_{\mathrm{BH}}}{M_{\mathrm{c}}}\right)^{3 / 4} r_{\mathrm{c}}
$$

the evolution timescale of the binary becomes the thermal timescale of the core. Here, $M_{\mathrm{BH}}$ is the mass of a black hole, $M_{\mathrm{c}}$ is the mass of the core, and $r_{\mathrm{c}}$ is the core radius. When the separation between the black holes becomes smaller than this $r_{\text {lc }}$, the binary effectively sweeps out the stars that can interact with the binary. Thus the evolution timescale becomes the thermal timescale of the core. They concluded that the black hole binary in the merger remnant has a lifetime much longer than the Hubble time.

Ebisuzaki et al. (1991) pointed out that the lifetime is much shorter if the BH binary is highly eccentric. BBR assumed that the binary remains circular. The timescale of the gravitational radiation is proportional to $(1-e)^{-3.5}$ for $e \sim 1$, where $e$ is the eccentricity. If the $\mathrm{BH}$ binary becomes highly eccentric, therefore, the lifetime would become much shorter. In fact, even for the average "thermal" eccentricity of 0.7 , the lifetime is shorter than that of a circular binary by nearly two orders of magnitudes. The binary evolves through the dynamical friction from field particles. If we naively apply Chandrasekhar's dynamical friction formula, the eccentricity should grow quickly since the dynamical friction is inversely proportional to the third power of the velocity, and therefore the strongest at the apoastron (Fukushige et al. 1992).

Mikkola and Valtonen (1992) numerically integrated the evolution of a BH binary in the core of a galaxy. They followed the orbit of BHs in the distribution of the field stars. The gravitational interaction between $\mathrm{BHs}$ and those between field stars and $\mathrm{BHs}$ are directly calculated. The interaction between the field stars is expressed by the force from a fixed potential. As a result, they could not follow the evolution of the structure of the core. However, they could use a fairly large number of particles $(N=10,000)$. They found that the binary hardened linearly in time. In other words, in their calculation the loss-cone depletion did not take place. For the eccentricity, what they found is that the eccentricity increases only very slowly. The rapid increase of the eccentricity predicted by Fukushige et al. (1992) did not take place. This is not very surprising because the standard dynamical friction formula used by Fukushige et al. (1992) is valid only for 
the motion of a single massive particle, and therefore is not guaranteed to give a correct result for the evolution of a binary. They also determined the rate of evolution of energy and eccentricity of the $\mathrm{BH}$ binary through scattering experiment. The result of their scattering experiment was consistent with the result of simulation.

Quinlan (1996) performed extensive scattering experiments of a BH binary and a field particle. He extended the work of Mikkola and Valtonen (1992) to include unequal-mass black holes. His result is similar to that of Mikkola and Valtonen (1992). The change in the eccentricity is small unless it is initially close to unity.

Makino et al. (1993) made self-consistent $N$-body simulation of the evolution of a BH binary in the core of a galaxy with 16,384 particles. Their results are summarized as follows: (a) The BH binary becomes harder beyond the loss-cone radius, without showing any sign of slowing down at least to $0.1 r_{\mathrm{lc}}$, where simulations were stopped. (b) After the binary is formed, the eccentricity of the binary remains roughly constant. (c) The eccentricity depends strongly on the initial condition of the BHs. Thus, their result is again consistent with the result of Mikkola and Valtonen (1992), but apparently in contradiction with the theoretical prediction of BBR.

In the present paper, we give the result of self-consistent direct $N$-body simulations with the number of particles much larger than that was employed in Makino et al. (1993) or Mikkola and Valtonen (1992).

In order to study the evolution of the binding energy of the central $\mathrm{BH}$ binary, we performed the merging simulations from the same initial condition, but with several different number of particles. The small- $N$ effect decreases the evolution timescale of the BH binary by at least two different ways. The first is that the timescale of the repopulation of the loss cone is proportional to the two-body relaxation time of the core. Therefore, if the timescale of the evolution of the binding energy is actually determined by the repopulation timescale, it should be proportional to the core relaxation time. In $N$-body simulations, however, the core relaxation time is not much larger than the timescale of the depletion itself. If the repopulation is faster than the depletion, the growth rate of the $\mathrm{BH}$ binary would be independent of $N$.

The other reason is that the center of mass of the $\mathrm{BH}$ binary has a small random velocity, since its kinetic energy is in equipartition with those of field particles (Bahcall and Wolf 1976, Mikkola and Valtonen 1992). Thus the BH binary might wander in, or even to outside, the loss cone. As a result, the effective radius of the loss cone might become larger in $N$-body experiments. The random velocity would be proportional to the square root of the mass ratio between the field particles and a BH binary. Therefore, a BH binary in the numerical simulation has the random velocity several hundred times larger than that of a real BH binary. Note that this second problem exists even in simulations with fixed field potential such as performed by Mikkola and Valtonen (1992), since in these calculations BHs feel the forces from the field particles, which shows the same fluctuating behavior as in self-consistent $N$-body calculations. Moreover, the effect of the relaxation is not completely suppressed either, because the orbits of field particles are changed by 
the interaction with the BH particles as well. Though the field particles do not interact directly with one another, they can still indirectly interact through interaction with BH particles.

In section 2 , we describe the numerical method we used. In section 3 , we describe the result of the simulations with different numbers of particles. Our main result is that the evolution timescale of the binary depends on the total number of field particles, i.e., the ratio between the mass of $\mathrm{BH}, M_{B H}$ and the mass of field particles, $m_{\text {field }}$. The relation between the timescale of the evolution of the binding energy of the $\mathrm{BH}$ binary, $t_{b}$, and the mass ratio between the $\mathrm{BH}$ and field particles is expressed as

$$
t_{b} \propto\left(\frac{M_{B H}}{m_{\text {field }}}\right)^{0.3},
$$

after the separation of two BHs reached a critical value which depends on the initial structure of the core. While the separation is larger than this critical value, the evolution timescale is almost independent of $N$. This critical value can be qualitatively understood as the loss cone radius, but is much smaller than the prediction of equation (1). The more sophisticated treatment by Quinlan (1996) seems to give better understanding.

For the timescale, a naive theoretical estimate based on the relaxation timescale should give $t_{b} \propto\left(M_{B H} / m_{\text {field }}\right)$ after the separation becomes smaller than the critical (loss-cone) separation. Section 4 is for discussions.

\section{NUMERICAL METHOD AND INITIAL CONDITION}

\subsection{NUMERICAL METHOD}

The calculation code we used is NBODY1 Aarseth 1985) modified to be used with GRAPE-4 special purpose computer for gravitational $N$-body simulation (Taiji et al. 1996). The time integration scheme is changed to the Hermite scheme (Makino and Aarseth 1992) to take advantage of the GRAPE-4 hardware. We neglect relativistic effects and treated BH particles as massive Newtonian particles. This treatment is good as long as the periastron distance of the BH pair does not become very small. Our simulations were terminated well before the relativistic effect would become important. We used a softened potential with the softening of $1 / 1024$ for the force between field particles, and pure $1 / r$ potential for forces to and from $\mathrm{BH}$ particles. The relative accuracy of the force calculated on GRAPE-4 is about 7 digits (IEEE single precision), which is more than enough for forces from field particles but might not be sufficient for the force from $\mathrm{BH}$ particles. In the present code, the force from field stars are calculated on GRAPE-4, while that from $\mathrm{BH}$ particles are calculated on the host computer in full double precision.

Calculations were performed on one cluster of GRAPE-4 system with the theoretical peak speed of 270 Gflops. Actual sustained speed was around 100-150 Gflops for simulations with $128 \mathrm{~K}-256 \mathrm{~K}$ particles. The $256 \mathrm{k}$ run took about five CPU days. 


\subsection{Initial Conditions}

The procedure to prepare the initial condition is the same as described in Makino and Ebisuzaki (1996, hereafter referred to as Paper I). We used the King model with the nondimensional central potential $W_{c}$ of 7 as the initial galaxy model. The number of particles is 2048 to 262144 . The initial galaxy is created so that the total mass $M_{g}$ is one and the total energy $E_{g}$ is $-1 / 4$ in the system of units where gravitational constant $G$ is one (the standard unit, Heggie and Mathieu 1986). The mass of a field particle is $m_{\text {field }}=1 / N$. The half mass radius of the initial galaxy model is about 0.75 and the half mass crossing time is $2 \sqrt{2}$. To place the central $\mathrm{BH}$, we removed $M_{B H} / m_{\text {field }}$ particles closest to the center of the galaxy at time $t=0$ and put the BH particle at the center. Thus the galaxy do not initially have a strong central cusp. In Paper I, we found that the structure of the merger does not depend on the details of the procedure to place the $\mathrm{BH}$ particles. For all calculations, $M_{B H}=1 / 32$ unless otherwise specified.

The initial orbit of two galaxies is parabolic with the periastron distance equal to 1 . The initial separation of two galaxies is 10 . We integrated the system to time $t=60$.

\section{Results}

\subsection{The evolution of the BH binary.}

Figure 1 shows the time evolution of the energy of the BH binary, $E_{b}$, for all runs. The energy $E_{b}$ is defined as

$$
E_{b}=\frac{1}{2} \mu v_{b}^{2}-\frac{M_{B H}^{2}}{r_{b}}=-\frac{M_{B H}^{2}}{2 a},
$$

where $\mu=M_{B H} / 2$ is the reduced mass of the $\mathrm{BH}$ binary, $r_{b}$ and $v_{b}$ are the relative distance and velocity of the two $\mathrm{BHs}$, and $a$ is the semi-major axis of the $\mathrm{BH}$ binary.

Before two galaxies merge, BHs lie at the centers of galaxies. Thus the distance is large and the binding energy of two BHs is negligible. In this period, $E_{b}$ is roughly equal to the sum of the kinetic energies of two BHs. The energy $E_{b}$ takes a maximum around $t=13$ because at that time two galaxies pass the periastron. The relative velocity of two galaxies is the largest at the periastron. Therefore $E_{b}$ takes the maximum value. By $t \simeq 30$, the second fallback takes place and two galaxies merge. Soon after that, two BHs form a binary.

From figure 1 we can see that the hardening rate of binaries, $-d E_{b} / d t$, is quite different for runs with different number of particles. The hardening rate is smaller for larger $N$. If we investigate figure 1 more closely, it seems the difference in the hardening rate is larger for the later time. Until $E_{b}$ reaches around -0.05 , the growth rate seem to be similar for all runs except for $N=2048$. In the 2048-body run, one of the BH particles had formed a bound pair with a field particle before it has become bound with another BH. When two BHs became bound, this field particle was still bound to a $\mathrm{BH}$ particle. This particle was bound to the BH binary until $t=40$. 
This is the reason why the curve for the 2048-body run is very noisy.

In order to see the dependence of the hardening rate on the energy itself, we plotted the hardening rate $-d E_{b} / d t$ as a function of $N$ in figure 2 . Here, $-d E_{b} / d t$ is calculated for two intervals of $E_{b},(-1 / 160,-1 / 80)$ and $(-1 / 10,-1 / 5)$. Note that for some runs $E_{b}$ at the end of the run shown in figure 1 did not reach $-1 / 5$. For these runs, we extended simulation so that $\left|E_{b}\right|>0.2$ at the end of the run.

Here we can clearly see that the hardening rate at the early phase the evolution seems to converge to $-d E_{b} / d t \sim 0.008$ for large $N$, while that for later phase of the evolution shows the power-law like behavior expressed roughly as $d E_{b} / d t \propto N^{-1 / 3}$ for the entire range of $N$.

Figure 3 shows the evolution of $E_{b}$ for repeated $256 \mathrm{k}$ runs. Here, the final merger of one run is used as the initial galaxy of the next run, following the procedure described in Paper I. In these runs, the central density of the initial galaxy becomes somewhat higher as we repeat the merging process. (See figure $4 \mathrm{~d}$ for the density profiles). Since the timescale of the evolution of the binary is proportional to the central density, this difference in the central density resulted in the difference in the growth timescale. This difference in the central density also resulted in the difference in the critical value of $E_{b}$.

There is no reason to assume that the first merger would give a reasonable result for the timescale, since our choice of the initial model is rather arbitrary. The structure of the core in the last merger events would be a more appropriate model for the initial galaxy. In this case, the critical value for $E_{b}$ seems to be around -0.3 .

\subsection{The structure of the core}

Figure 4 shows the density profiles of the central region of the merger remnant from $256 \mathrm{k}, 32 \mathrm{k}$ and $4 \mathrm{k}$ runs for different times. For the $256 \mathrm{~K}$ run, the density is almost flat at the very central region, with possible minimum at $r \sim 0.01$, where $r$ is the radius. For $32 \mathrm{k}$ runs, the central region

has a cusp of roughly $\rho \sim r^{-0.5 \sim-1}$, and for $4 \mathrm{k} \rho \sim r^{-1 \sim-1.5}$. For all runs, central density decreases in time.

The difference in the central structure explains the difference in the growth rate of $E_{b}$ shown in figures 1 and 2. The growth rate is proportional to the stellar mass density around the $\mathrm{BH}$ binary. For higher $N$ the density is lower. Therefore growth rate is smaller for larger $N$.

The difference in the structure of the core is due to the difference in the central relaxation time. Since the merger remnant does not have a flat core, the central relaxation time is rather difficult to define. We calculated the local relaxation time as the function of the radius $r$, and found that it takes the minimum value around $r=0.1$ for all models. So we regard this minimum value as the central relaxation time, $t_{r c}$. At time $t=40, t_{r c}$ is around 200,800 and 5000 for $4 \mathrm{k}$, $32 \mathrm{k}$ and $256 \mathrm{k}$ runs, respectively. Thus, for the $4 \mathrm{k}$ run the simulation timespan is comparable to 
the relaxation time, while for the $256 \mathrm{k}$ run the relaxation timescale is orders of magnitudes larger than the simulation timespan.

The flat central region observed in the $256 \mathrm{k}$ run is a kind of the loss cone predicted by BBR. In BBR's theory, when the binary becomes sufficiently hard, it kicks out all the stars it can interact and then the growth slows down significantly.

It, however, should be noted that the structure we observed is rather different from BBR's picture or the standard model for the star cluster with massive central BH (Shapiro 1985, and references therein), which predict the cusp with $\rho \propto r^{-1.75}$ outside a few times the radius of the $\mathrm{BH}$ binary. For the $256 \mathrm{k}$ run, the numerical result is a very shallow cusp outside the loss cone like $\rho \propto r^{-0.5}$. For runs with smaller $N$ the slope is steeper. Thus, it seems the cusp becomes shallower as we increase $N$. On the other hand, the theoretical prediction is a universal cusp of $\rho \propto r^{-1.75}$. We will discuss possible interpretations in section 4 .

Figure 5 gives the surface brightness profiles for same runs as in figure 4. Here, the time evolution is not as pronounced as in figure 3. In addition, we cannot see any decrease of the luminosity toward the center even for the $256 \mathrm{k}$ run.

\section{Summary and Discussion}

We performed the simulation of the evolution of massive BH binaries formed through mergings of ellipticals by means of direct $N$-body simulations. Our major findings are summarized as follows.

a) The timescale of the evolution of the binary depends on the total number of particles, in other words, the mass ratio between BHs and field particles. However, the dependence is very weak for the early phase of evolution. For later phase, we observed $t_{b} \propto N^{1 / 3}$, which is much weaker than the prediction of the theoretical model.

b) The evolution timescale shows very strong dependence on the initial central density.

c) For large $N$ runs, the "loss cone" depletion effect is clearly visible. In other words, the stellar density around the $\mathrm{BH}$ is lower for larger $N$. However, again, the density profile we obtained for simulations with large $N$ was quite different from the theoretical model which gives the density cusp of $\rho \propto r^{-1.75}$.

In the following, we discuss the implication of these results in some details. 


\subsection{The hardening rate of the $\mathrm{BH}$ binary}

For the early phase of the evolution, it is not surprising that the evolution timescale is independent of $N$. This is because the loss cone depletion has not taken place. However, our result shows that the the loss-cone radius is much smaller than the prediction by BBR.

This difference might be because of BBR's assumption in calculating the loss-cone radius. They assumed that the loss-cone depletion occurs when the total kinetic energy of all stars which can interact with the binary becomes smaller than the binding energy of the binary. Quinlan (1996) argued that the loss-cone depletion occurs only when all particles that can interact with the BH binary are ejected out of the core. In his examples, this assumption leads to the loss-cone radius a few times smaller than the semi-major axis for the " $1 k T$ " binary (the binary with the orbital velocity comparable to the typical velocity of field stars). His result is qualitatively consistent with our present result. For our runs, the critical value of $E_{b}$ is $-2 \sim 10 \cdot M_{B H} v_{c}^{2}$, depending on the density profile, where $v_{c}$ is the r.m.s. velocity of field particles.

After the $\mathrm{BH}$ reaches the loss cone radius, its evolution slowed down. However, the dependence of the growth timescale on $N$ is much weaker than the theoretical prediction of $t_{b} \propto N$. This difference might be understood by means of the detailed treatment of the evolution of the stellar distribution around the central black hole developed by Shapiro and his collaborators Shapiro 1985, Duncan and Shapiro 1983).

The essence of their argument is that the stars are removed from the system only when it actually come close enough to the BH (they considered the distribution of stars around a single $\mathrm{BH})$. Thus, if the average change of the angular momentum of a typical star in the core in one orbital period is larger than the maximum angular momentum of the star in the loss cone orbit, the loss cone is repopulated in the timescale shorter than the depletion timescale. In this case, the dependence of the growth timescale on $N$ might be weaker than the theoretical prediction.

The maximum angular momentum of a field particle, $J_{\max }$, that can interact with the $\mathrm{BH}$ binary is expressed roughly as

$$
J_{\max } \sim \sqrt{M_{B H} a} \sim 0.01 E_{b}^{-1 / 2},
$$

where $a$ is the semi-major axis of the BH binary an we used $M_{B H}=1 / 32$.

On the other hand, the average change in the angular momentum of a typical star in the core per orbit is given roughly by

$$
\Delta J \sim r_{c} v_{c} \sqrt{t_{c} / t_{r c}}
$$

where $r_{c}, v_{c}, t_{c}$ and $t_{r c}$ are the core radius, the r.m.s. velocity of the particles in the core, the

crossing time and the relaxation time of the core, respectively. If we use $r_{c}=0.1$ and $N_{c}=N / 20$ as a typical value for our simulation (see figure 4), we have

$$
\Delta J \sim \sqrt{\frac{2 \log (0.02 N)}{N}} .
$$


For $E_{b} \sim-0.1, \Delta J>J_{\max }$ in the case of $N=2048$ and the opposite in the case of $N=262144$. Thus, the range of $N$ in our simulation just cover the transition from $J_{\max }<\Delta J$ to $J_{\max }>\Delta J$. It is not at all surprising that the dependence of the growth rate is weaker than the prediction of the theory which assumes $J_{\max }>>\Delta$. In order to really determine the dependence of the lifetime on $N$, we need to employ more particles.

Simulations with number of particles larger than used here might be possible by means of more approximate schemes such as the treecode with individual timesteps (McMillan and Aarseth 1993) or an extension of the SCF method Quinlan et al. 1995) to the case of central BH binary.

It should be noted that here we study the thermal evolution of the system since if the system is collisionless the loss cone will never be repopulated. Thus, the two-body relaxation effect should be modeled appropriately. The use of the so-called "collisionless" scheme should be done very carefully.

\subsection{The evolution of $\mathrm{BH}$ binaries in real ellipticals}

Here we discuss the evolution of $\mathrm{BH}$ binaries in real ellipticals. For simplicity, we assume that the evolution of the binary stops at the critical energy obtained by our experiments.

The timescale of the gravitational radiation is given by

$$
t_{\mathrm{GR}}=2 \times 10^{15} g(e)\left(M_{\mathrm{BH}} / 10^{8} M_{\odot}\right)^{-3}(a / 1 \mathrm{pc})^{4} \quad \mathrm{yr},
$$

with $g(e)$ given by

$$
\begin{aligned}
g(e) & =\frac{\left(1-e^{2}\right)^{7 / 2}}{1+(73 / 24) e^{2}+(37 / 96) e^{4}} \\
& \simeq 2(1-e)^{7 / 2} \quad(e \sim 1) .
\end{aligned}
$$

which, in our unit,

$$
t_{\mathrm{GR}}=5 \times 10^{9} g(e)\left(M_{\mathrm{BH}} / 10^{8} M_{\odot}\right)\left(v_{c} / 300 \mathrm{~km} / \mathrm{s}\right)^{-8} E_{b}^{-4} \quad \mathrm{yr} .
$$

Thus, the lifetime of the BH binary with $E_{b}=-0.05$ is $\sim 10^{15}$ years, which is well over the Hubble time. For $E_{b}=-0.3$, the lifetime is more than $10^{11}$ years. Unless the eccentricity is relatively large, it is unlikely for the BH binary to merge.

BBR (see also Rees 1990) argued that if the BH binary does not merge in the time significantly shorter than the Hubble time it is likely to be kicked out from the core or the galaxy itself when the parent galaxy merges with yet another galaxy, or at least kicks out the third binary, through the gravitational slingshot.

However, the slingshot mechanism is not as effective as assumed by BBR. When one BH binary and a single $\mathrm{BH}$ reside in the core, there are two possible outcomes. In one case, one of the 
three BHs is ejected out of the parent galaxy through the slingshot. In the other case, the binary merges through gravitational radiation before kicking out the third BH. BBR concluded that the slingshot is the likely outcome, assuming that the binary is circular. Makino and Ebisuzaki (1995) showed that the slingshot is unlikely to occur if the distribution of the eccentricity is correctly taken into account. They showed that the typical lifetime of the BH binary before the ejection of the third body is less than $10^{9}$ years. If the $\mathrm{BH}$ binary merged before the third $\mathrm{BH}$ is ejected out of the galaxy, the final state is the binary of the merged $\mathrm{BH}$ and the third $\mathrm{BH}$.

Thus, it is quite possible that a large fraction of ellipticals have central $\mathrm{BH}$ binaries. The implication of the presence of the binary was addressed briefly in BBR. Of course, if the velocity dispersion is higher, the timescale of the gravitational radiation at the loss cone separation become much shorter. The opposite is also true. If $v_{c}<200 \mathrm{~km} / \mathrm{s}$, the mechanism described in Makino and Ebisuzaki (1995) cannot make the BH binary merge before it eject the third body. On the other hand, if $v_{c}>600 \mathrm{~km} / \mathrm{s}$, the BH binary would merge through the dynamical friction and the gravitational radiation. Since most of ellipticals fall in the range of $200 \mathrm{~km} / \mathrm{s}<v_{c}<600 \mathrm{~km} / \mathrm{s}$, the fate of the $\mathrm{BH}$ binary is difficult to predict. Interaction with gas might determine the final fate.

If triple $\mathrm{BH}$ systems are not very uncommon, we might be able to observe some of them as the QSO activities located far from the center of the galaxy. Recent HST observations of nearby QSOs (Bahcall, Kirhakos, and Schneider 1995a, 1995b, 1995c) suggested many of the QSOs are not located at the center of the galaxy. In the triple $\mathrm{BH}$ system, BHs would spend most of the time in the halo "parking" orbit, as in the case of the binaries in globular clusters Hut et al. 1992). Thus, QSO activities might be observed in the positions far from the center of the parent galaxy. To explain QSOs outside the core of the parent galaxy, Fukugita ant Turner (1996) proposed a scenario in which the massive BH are formed independently from the galaxies. Such an extraordinary model might not be necessary if triple BHs are common.

\subsection{The structure of the central region}

Our numerical result for the density profile around the BH pair is quite different from the standard picture of the cusp with $\rho \propto r^{-7 / 4}$. Though the cusp exists, its slope is smaller for large $N$.

For large- $N$ runs, it is quite natural that the slope of the cusp is different from the theoretical prediction, simply because the duration of the simulation is much shorter than the thermal relaxation time of the core. It takes the thermal relaxation time of the core for the cusp to fully develop. For the $256 \mathrm{k}$ run, the local two-body relaxation time at $r=0.1$ is $5 \times 10^{3}$ for $t=40$. Thus the cusp cannot develop fast enough.

Since the two-body relaxation has even smaller effect in real ellipticals than in our largest simulations, we can safely predict that the $r^{-7 / 4}$ cusp does not present in ellipticals with central

massive BH. The structure is most likely to be a cusp of $r^{-0.5}$ formed by the particles not bound 
to the BH binary (Duncan and Shapiro 1983), terminated at the loss cone radius. This is in good agreement with the resent HST observations of the core of large ellipticals which shows the cusp with the slope $-0.5 \sim-1$. This shallow cusp indicates that the central $\mathrm{BH}$ is placed in the galaxy in dynamical timescale. If the $\mathrm{BH}$ mass grows in the timescale longer than the dynamical time, the slope would be -1.5 (Young 1980).

I'm grateful to Gerry Quinlan for his critical comments on the very early version of this paper and stimulating discussions. I also thank Piet Hut for helpful discussions, Yoko Funato and Toshiyuki Fukushige for comments on the manuscript. This work was supported by Grant-in-Aid for Specially Promoted Research (04102002) of the Ministry of Education, Science and Culture, Japan. 


\section{REFERENCES}

Aarseth, S. J., 1985, in Multiple Time Scales, ed. J. U. Brackhill and B. I. Cohen (Academic Press, New York), 377.

Bahcall, J. N., Kirhakos, S., \& Schneider, D. P., 1995, ApJ 435, L11.

Bahcall, J. N., Kirhakos, S., \& Schneider, D. P., 1995, ApJ 450, 486.

Bahcall, J. N., Kirhakos, S., \& Schneider, D. P., 1995, ApJ 447, L1.

Bahcall, J. N. \& Wolf, R. A., 1976, ApJ 209, 214.

Begelman, M. C., Blandford, R. D., and Rees, M, J. 1980, Nature, 287, 307.

Duncan, M. J. \& Shapiro, S. L. 1983, ApJ 268, 565.

Ebisuzaki, T., Makino, J., and Okumura, S. K., 1991, Nature, 354, 212.

Fukugita, M. \& Turner, E. 1996, ApJ, 460, L81.

Fukushige, T., Ebisuzaki, T. and Makino, J., 1992, PASJ, 44, 281.

Heggie, D.C. \& Mathieu, R.D., 1986, in The Use of Supercomputers in Stellar Dynamics, eds. P. Hut and S. McMillan [Springer], p. 233.

Hut, P., McMillan, S. L. W., \& Romani, R. W. 1992, ApJ, 389, 527.

Lauer, T. R., Ajhar, E. A., Byun, Y. I., Dressler, A. Faber, S. M., Grillmair, C., Kormendy, J. Richstone, D. \& Tremaine, S. 1995, AJ, 110, 2622.

Makino, J. and Aarseth, S. J., 1992, PASJ, 44, 141.

Makino, J., Fukushige, T., Okumura, S. \& Ebisuzaki, T. 1993, PASJ, 45, 303.

Makino, J. \& Ebisuzaki, T. 1995, ApJ, 436, 607.

Makino, J. \& Ebisuzaki, T. 1996, ApJ, 465, xxx.

McMillan, S. L. W. and Aarseth, S. J., 1993, ApJ, 414, 200.

Mikkola, S. \& Valtonen, J. 1992, MNRAS, 259, 115.

Rees, M. J., 1990, Science, 247, 817.

Shapiro, S. L. 1985, in Dynamics of star clusters, IAU Symposium No. 113, eds. P. Hut \& J. Goodman, Dordrecht, D. Reidel Publishing Co.

Quinlan, G. D., Hernquist, L. \& Sigurdsson, S. 1995, ApJ, 440, 554. 
Quinlan, G. D., 1996, preprint.

Taiji, M., Makino, J., Fukushige, T., Ebisuzaki, T. \& Sugimoto, D. 1996, in Dynamical evolution of star clusters, IAU Symposium No. 174, eds. J. Makino and P. Hut (in press).

Valtonen, J., Mikkola, S., Heinamaki, P. \& Valtonen, H. 1994, ApJS 95, 69.

Young, P. 1980, ApJ, 242, 1232. 


\section{Figure Captions}

Fig. 1. - The time evolution of the energy of the BH binary for runs with $N=2 \mathrm{k}$ (bottom) to $256 \mathrm{k}$ (top). Thin curves represent $2 \mathrm{k}$ (solid), 4k (short dashed), 8k (long dashed) and 16k (dot dashed) runs. Thick curves represent 32k (solid), 64k (short dashed), 128k (long dashed) and 256k (dot dashed) runs.

Fig. 2.- The growth rate of the binary binding energy $-d E_{b} / d t$ plotted versus the total number of particles $N$. Open and filled circles the average rate for $-1 / 160>E_{b}>-1 / 80$ and $-1 / 10>E_{b}>-1 / 5$, respectively.

Fig. 3. - Same as figure 1 but for repeated mergers from $\mathrm{N}=256 \mathrm{k}$ run. Solid, dotted, short-dashed, long-dashed and dot-dashed curves represent 1st through 5th mergers, respectively.

Fig. 4.- Density profiles of the central region of the mergers. (a-c) Runs with (a) 256k, (b) $32 \mathrm{k}$, and (c) $4 \mathrm{k}$ particles. Open circle, filled squares and filled circles are the profiles at $t=40,50$ and 60, respectively. (d) Density profiles of repeated mergings. Filled circles, filled squares, filled triangles, open circles and open squares represent the profiles for 1st through 5th mergers. For all profiles, $t=40$. Initial total number of particles is $256 \mathrm{k}$.

Fig. 5.- Same as figure 4 but for surface density profiles. 


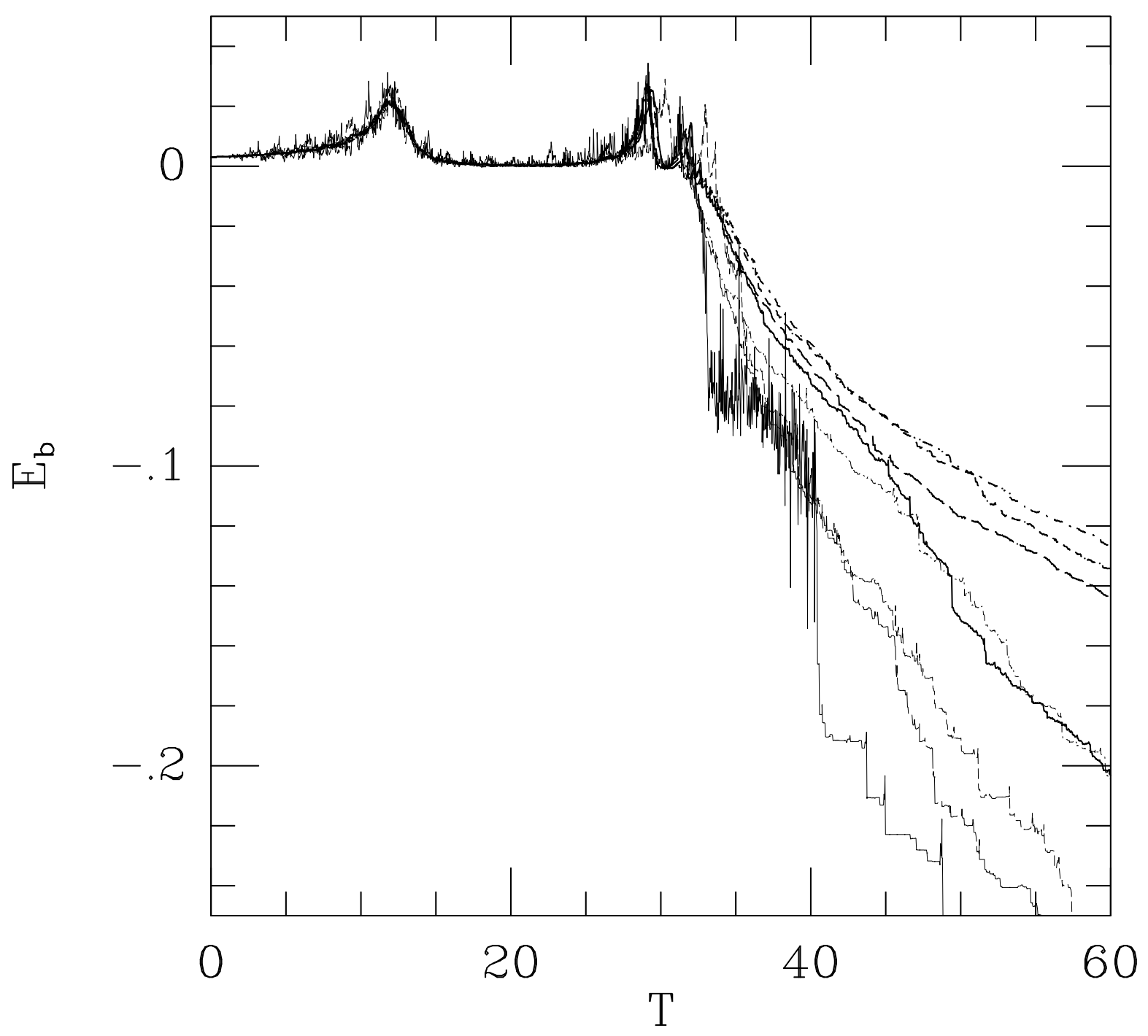

Figure 1 


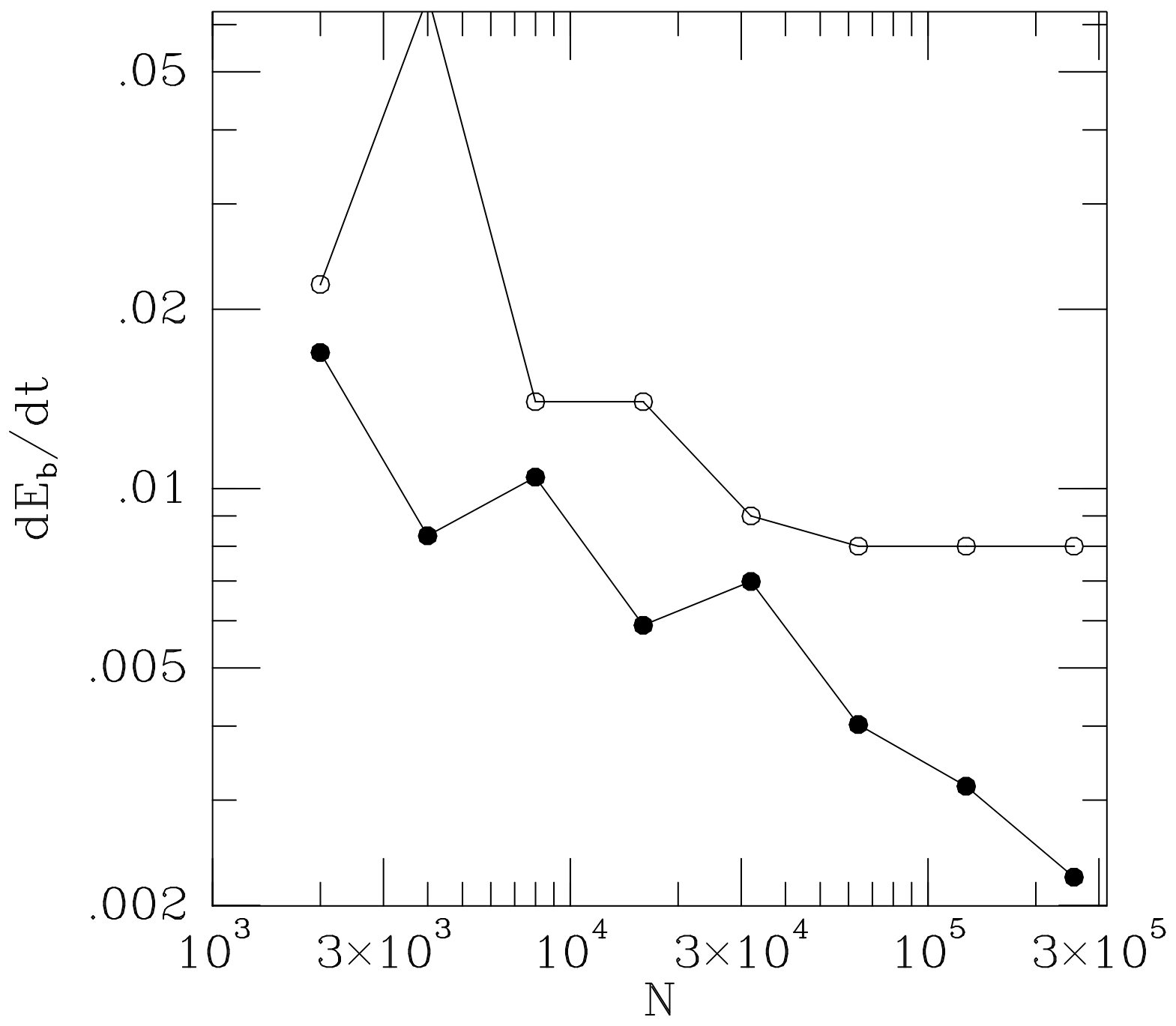

Figure 2 


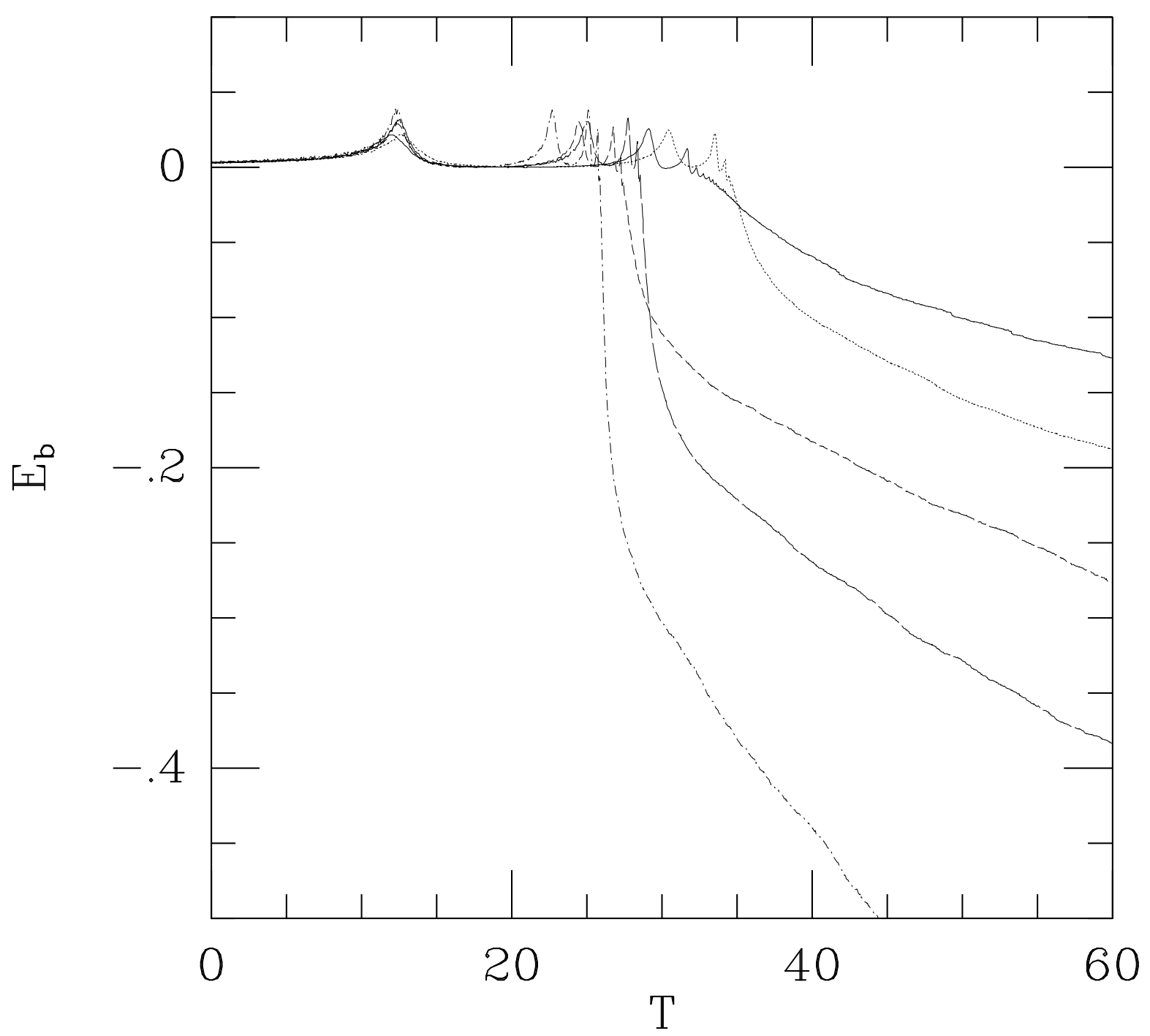

Figure 3 

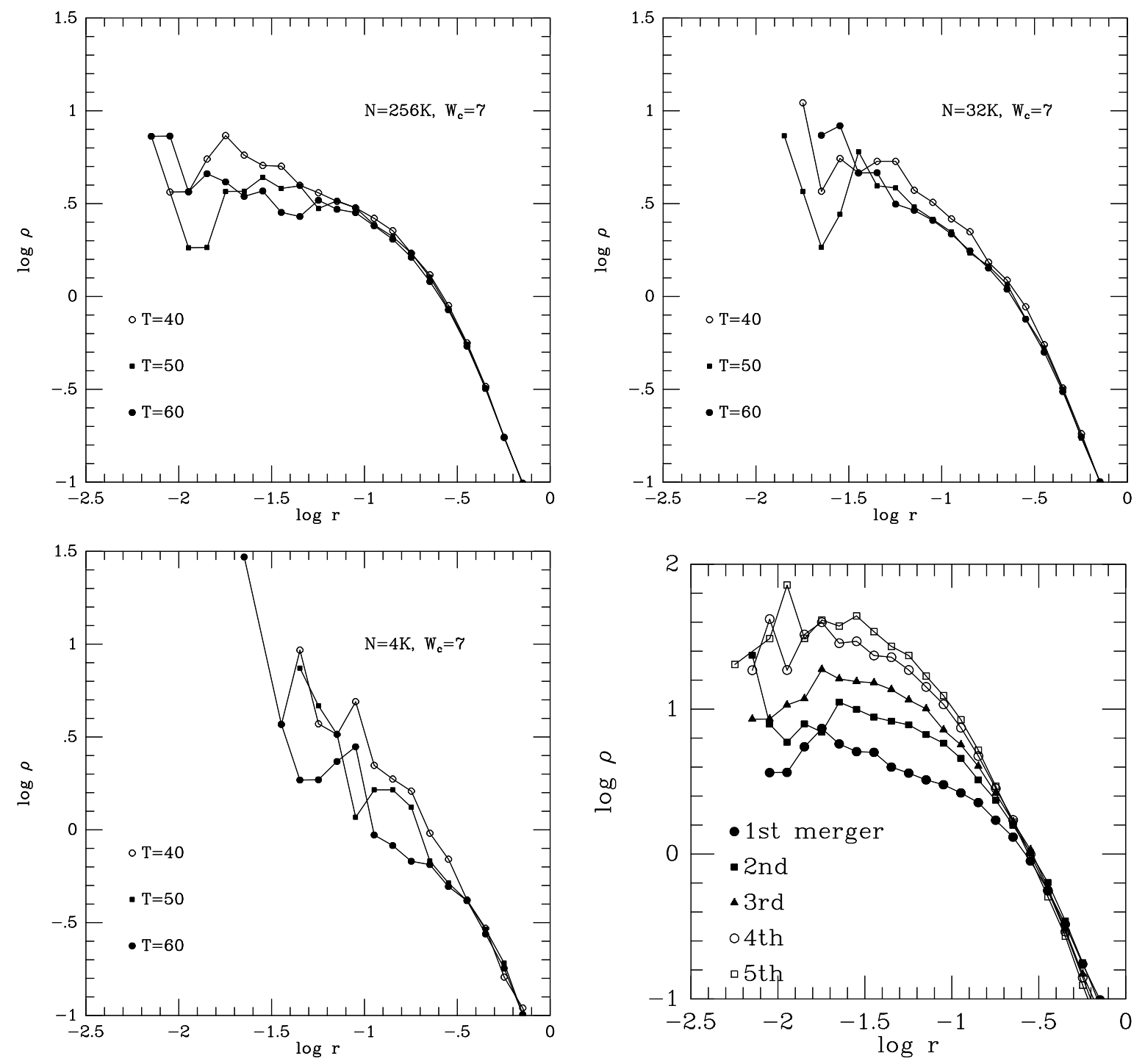

Figure 4 

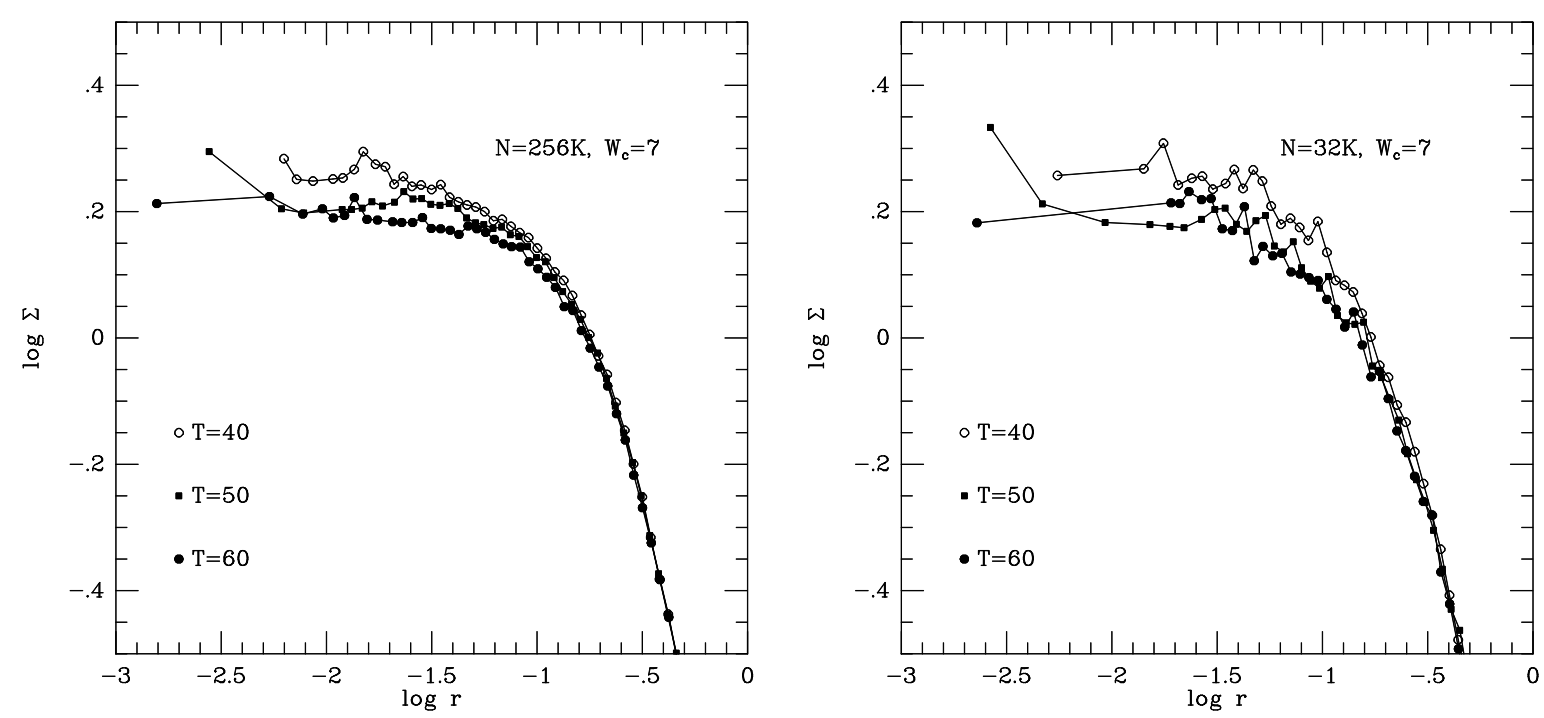


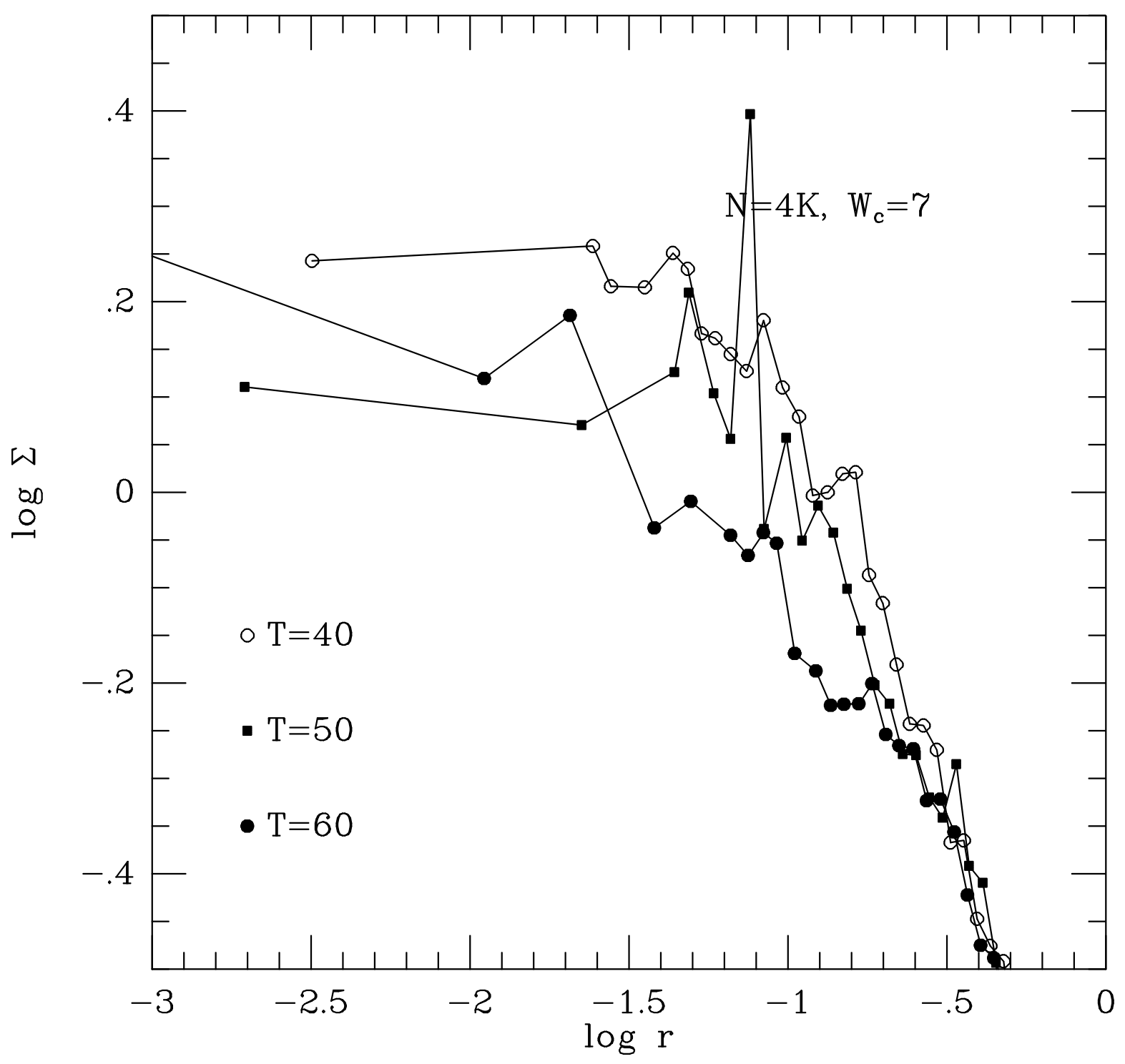

Figure 5 